\title{
Estudio de la temperatura de crecimiento sobre la cristalinidad en películas delgadas de $\mathrm{BaTiO}_{3}$
}

\section{Substrate Temperature Study in the Crystallinity of $\mathrm{BaTiO}_{3}$ Thin Films}

\author{
Márquez-Herrera Alfredo \\ Coordinación Académica Región Altiplano (COARA) \\ Universidad Autónoma de San Luis Potosí \\ Correo:alfredo.marquez@uaslp.mx \\ Hernández-Rodríguez Eric Noé \\ Centro de Investigación en Ciencia Aplicada y \\ Tecnología Avanzada \\ Unidad Legaría del Instituto Politécnico Nacional \\ Correo:noehmx@hotmail.com \\ Zapata-Torres Martín Guadalupe \\ Centro de Investigación en Ciencia Aplicada y \\ Tecnología Avanzada \\ Unidad Legaría del Instituto Politécnico Nacional \\ Correo:mzapatat@ipn.mx
}

\author{
Cruz-Jáuregui María de la Paz \\ Centro de Nanociencias y Nanotecnología \\ de la Universidad Nacional Autónoma de México \\ Correo:mcruz@cnyn.unam.mx
}

Meléndez-Lira Miguel Ángel

Departamento de Física

Centro de Investigación y de Estudios Avanzados

Instituto Politécnico Nacional

Correo:mlira@fis.cinvestav.mx

\section{Información del artículo: recibido: marzo de 2011, aceptado: septiembre de 2012}

\section{Resumen}

Películas delgadas Ferroeléctricas de $\mathrm{BaTiO}_{3}$ (BTO) se depositaron a partir de un blanco de $\mathrm{BaTiO}_{3}$ mediante la técnica de RF-Sputtering (erosión catódica por radio frecuencia) sobre substratos de nicromel y cuarzo. Se estudió el efecto de la temperatura de sustrato in-situ en la cristalinidad del material durante su depósito. Estas muestras fueron comparadas con películas depositadas a temperatura ambiente y tratadas térmicamente posterior al depósito fuera de la cámara de crecimiento. El estudio de la cristalinidad fue realizado mediante la técnica de difracción de rayos-X. Adicionalmente, se llevaron a cabo caracterizaciones ópticas mediante un espectrofotómetro UV-Vis. El crecimiento de películas delgadas con temperatura de sustrato permite la obtención de materiales cristalinos a temperaturas por debajo de las reportadas por otros autores.

\section{Descriptores:}

- películas delgadas

- tratamientos térmicos

- $\mathrm{BaTiO}_{3}$ 


\begin{abstract}
Ferroelectric thin films of $\mathrm{BaTiO}_{3}(\mathrm{BTO})$ were grown on quartz and nichrome substrates using a $\mathrm{BaTiO}_{3}$ target by RF-Sputtering technique. It was studied the effect of the substrate temperature in the crystallization of the material. These samples were compared with films deposited at room temperature and heat treated out of the growth Chamber. Their crystallinity were studied by X-ray diffraction. Additional$l y$, the optical characterizations were carried out by UV-Vis spectrophotometer. The growth of thin films with substrate temperature allows the obtaining of crystalline materials at temperatures below those reported by other authors.
\end{abstract}

\section{Introducción}

Las películas delgadas ferroeléctricas han sido motivo de muchas investigaciones en el mundo debido a sus aplicaciones en la microelectrónica. En particular, el titanato de bario $\left(\mathrm{BaTiO}_{3}\right.$ o simplemente llamado $\left.\mathrm{BTO}\right)$ en forma de película delgada es un material con una constante dieléctrica muy alta, una baja corriente de fuga y que además posee propiedades ferroeléctricas que pueden modularse mediante la adición de algún dopante (Pasierb et al., 1998), permitiendo una gran gama de aplicaciones, desde simples condensadores hasta complicados dispositivos microelectrónicos (Hirata et al., 1999; Shi et al., 2002; Ruckenbauer et al., 2004; Kirchoefer et al., 1998; Keis et al., 1998). Por otro lado, películas delgadas de $\mathrm{BaTiO}_{3}$ se han depositado por muchas técnicas como: rocío pirolítico, láser pulsado, sol-gel, MOVCD, etcétera (Pasierb et al., 1998; Ruckenbauer et al., 2004, Cheng et al., 2000; Somekh, 1984), pero la técnica de RF-Sputtering ha mostrado ser una de las más adecuadas para el depósito de este material, ya que permite preservar su estequiometría.

Por otra parte, independientemente de la técnica utilizada para la preparación de películas delgadas ferroeléctricas, y particularmente del $\mathrm{BaTiO}_{3}$, éstas se obtienen en estado amorfo y requieren de altas temperaturas para lograr su cristalización, ya que sólo en ese estado presentan sus propiedades ferroeléctricas. Para lograr su cristalización es necesario aplicarle tratamientos térmicos a temperaturas por arriba de los $450^{\circ} \mathrm{C}$ (Zhu et al., 2010), lo cual limita el uso en aplicaciones donde se aprovechan sus propiedades ferroeléctricas como en las memorias ferroeléctricas, sensores piezoeléctricos, piroeléctricos, etcétera. Además, el silicio juega un rol dominante en su integración en la industria electrónica, donde la heteroestructura $\mathrm{Pt} / \mathrm{TiO}_{2} / \mathrm{SiO}_{2} / \mathrm{Si}$ se usa comúnmente como un sustrato para las películas ferroeléctricas debido a que integra estos materiales con la tecnología del silicio, aunado a que permite tener buena estabilidad mecánica y térmica, baja resistencia eléctrica, alta resis- tencia a la oxidación y una excelente adherencia entre el BTO y el silicio (Hirata et al., 1999). Sin embargo, este sustrato presenta varios inconvenientes debido a las altas temperaturas empleadas en el proceso de tratamiento térmico para la cristalización del BTO, donde el titanio migra hasta la superficie del platino y añade resistencia eléctrica al electrodo (Sreenivas et al., 1994, Lee et al., 1995; Kim et al., 1997). En este trabajo se sugiere al nicromel como un sustrato alternativo para el estudio de la cristalinidad de las películas. Además de la gran cercanía que existe entre los picos de difracción correspondientes a la heteroestructura $\mathrm{Pt} / \mathrm{TiO}_{2} / \mathrm{SiO}_{2} / \mathrm{Si}$ y los picos originados por el BTO, se imposibilita su análisis; en cambio, los picos de difracción correspondientes al nicromel no coinciden con los del BTO, además de que este tipo de sustrato es fácil de obtener, de bajo costo y es un buen candidato sustituto del $\mathrm{Pt} / \mathrm{TiO}_{2} / \mathrm{SiO}_{2} / \mathrm{Si}$ (Márquez et al., 2005). Adicionalmente, las altas temperaturas empleadas para la cristalización del BTO producen grietas en el material debido al estrés térmico, por lo que una de las grandes necesidades en la obtención de estas películas es encontrar una metodología que permita obtener el material en forma cristalina, empleando bajas temperaturas de depósito para evitar los problemas antes mencionados. En este sentido, se diseñó y construyó un sistema de calentamiento económico y compacto para el sistema de depósito por RF-Sputtering, capaz de trabajar bajo condiciones de atmósfera reactiva, donde se puedan obtener películas uniformes, homogéneas y con propiedades cristalinas bien controladas. Un esquema general de dicho sistema se muestra en la figura 1.

Este sistema de tratamientos térmicos in-situ es capaz de proporcionar energía térmica al sustrato durante el proceso de crecimiento de las películas delgadas de BTO, propiciando de esta manera la cristalización del material en el proceso mismo de crecimiento. Los detalles del diseño y construcción de este sistema ya fueron reportados (Márquez et al., 2010).

Por lo anterior, en este trabajo se estudian las ventajas que presenta el tratamiento térmico in-situ en la cris- 


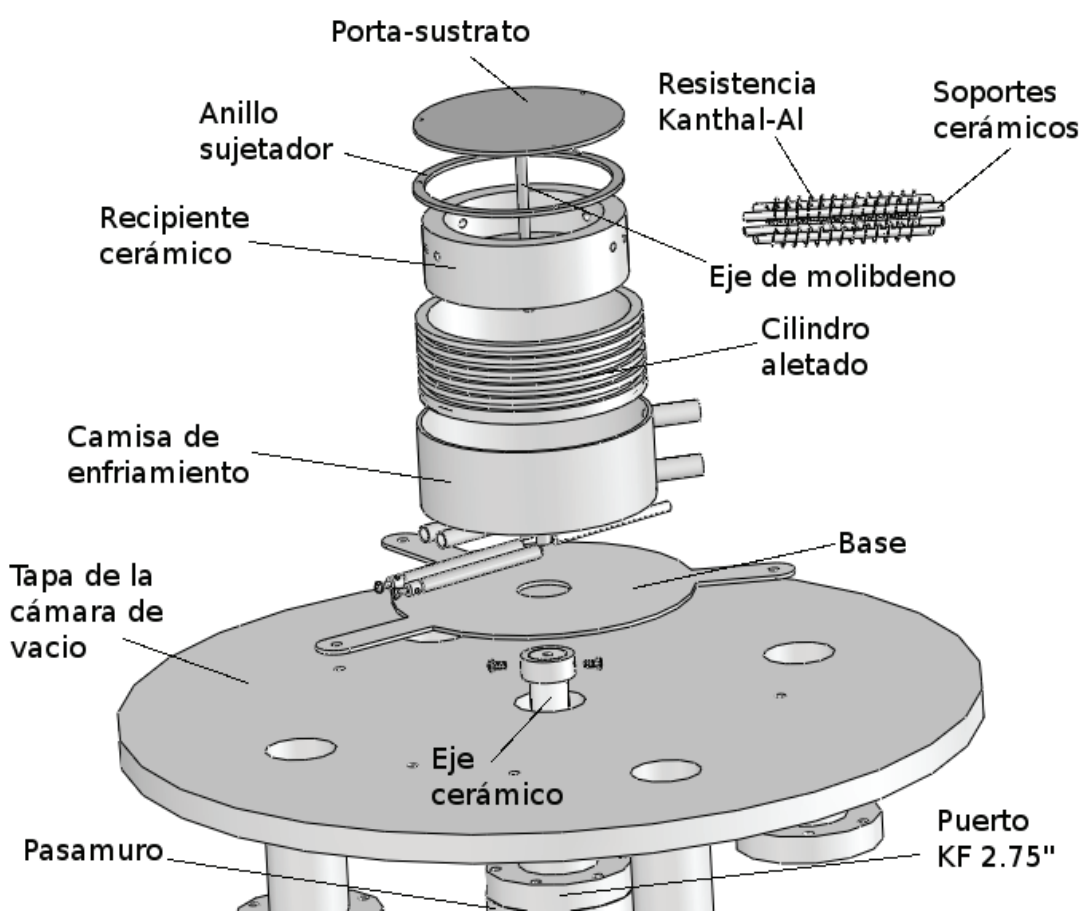

Figura 1. Esquema del sistema de calentamiento talinidad de las películas y su influencia en las propiedades ferroeléctricas contra los tratamientos térmicos, realizados fuera del propio sistema de depósito.

\section{Desarrollo experimental}

El crecimiento de las películas se realizó mediante la técnica de RF-Sputtering con magnetrón a partir de un blanco de BTO con un diámetro de $50.8 \mathrm{~mm}, 63 \mathrm{~mm}$ de espesor y $99.9 \%$ de pureza en un plasma $\mathrm{Ar} / \mathrm{O}_{2}$. Los detalles de la fabricación del blanco ya fueron reportados por Torres et al. (2005). El sustrato de nicromel fue obtenido de una cinta comercial, nicromel 80, con $0.127 \mathrm{~mm}$ de espesor de H. Cross Company, Inc.

Antes del depósito, los sustratos fueron limpiados en baño ultrasónico empleando acetona, metanol e isopropanol durante 15 minutos y después fueron enjuagados en agua desionizada para finalmente secarse con N2. Para llevar a cabo el depósito, la cámara de sputtering fue llevada a una presión de $5 \times 10^{-6}$ Torr o menos, después se realizó un arrastre de gases "flushing" con Ar a una presión de 30 mTorr durante 10 min. Posteriormente, se introdujo una mezcla de gases de Ar y $\mathrm{O}_{2}$ con una razón Ar: $\mathrm{O}_{2}$ de 90:10 y fue mantenido a una presión de 50 mTorr para encender el plasma bajo una potencia de $160 \mathrm{~W}$. Después, la presión fue reducida a 30 mTorr para hacer un pre-sputtering por $15 \mathrm{~min}$ para limpiar la superficie del blanco. Finalmente, el magne- trón fue abierto para iniciar el depósito del material en forma de película delgada hasta obtener un espesor de 365 nm. Para proveer una superficie uniforme, se empleó una geometría fuera de eje, "off-axis" de $30^{\circ}$ y el sustrato fue rotado a $100 \mathrm{rpm}$. Se prepararon dos series de muestras bajo la misma metodología. En la primera serie, las muestras fueron crecidas con temperatura de sustrato, mientras que en la segunda, las muestras fueron crecidas a temperatura ambiente. Posteriormente fueron tratadas térmicamente en un sistema externo.

En ambos casos, las temperaturas de tratamiento fueron $375^{\circ} \mathrm{C}, 435^{\circ} \mathrm{C}, 495^{\circ} \mathrm{C}$ y $549^{\circ} \mathrm{C}$ durante 1 hora. Con el objeto de evitar grietas en las películas, los tratamientos térmicos externos se hicieron con rampas de temperatura durante el calentamiento y el enfriamiento, que consistieron de pasos de $100^{\circ} \mathrm{C} / \mathrm{min}$, manteniendo por 10 minutos cada paso. Los detalles de su fabricación ya fueron reportados por Torres et al. (2005).

El espesor de las películas fue medido mediante un perfilómetro Dektak3ST marca Veeco Instruments al mismo tiempo que la microestructura de la superficie y la composición química de las muestras fueron evaluadas empleando un microscopio electrónico de barrido (MEB) JSM-5300 marca Jeol, equipado con un detector EDS (del inglés Electron Dispersive Spectroscopy). Los difractómetros de difracción de rayos-x se obtuvieron mediante el empleo de un difractómetro Philips X'pert empleando la línea $\mathrm{K} \alpha$ de un blanco de cobre operado a 
$40 \mathrm{kV}$ y $150 \mathrm{~mA}$ y la modalidad normal para polvos $\theta-2 \theta$. Los espectros de transmisión óptica de las películas se lograron mediante un espectrofotómetro UV-Vis Hitachi $3300 \mathrm{H}$. Para esta prueba las películas fueron depositadas sobre cuarzo para evitar la absorción del sustrato en el rango de 200-900 $\mathrm{nm}$.

\section{Resultados y discusión}

Las mediciones de perfilometría mostraron que el espesor obtenido en las películas delgadas de BTO es de alrededor de $365 \pm 10 \mathrm{~nm}$, mientras que el análisis por EDS de su composición química indicó que éstas son estequiométricas. En la figura 2 se muestran micrografías que se obtuvieron a través del MEB de las películas de BTO crecidas sobre sustratos de nicromel con temperatura de sustrato (figura 2a) y tratamiento térmico empleando un sistema externo (figura $2 b$ ), en ambos casos a $548.8^{\circ} \mathrm{C}$.

Como puede verse en la figura 2, aunque las películas fueron depositadas bajo las mismas condiciones, con excepción de la temperatura de sustrato, las morfologías son muy similares.

Por otra parte, en la figura 3 se muestran los espectros de difracción de rayos $\mathrm{X}$ de las películas de BTO, depositadas sobre sustratos de nicromel a temperatura ambiente y con tratamiento térmico externo de $375^{\circ} \mathrm{C}$ a $549^{\circ} \mathrm{C}$. En los difractogramas, los dos picos de alta intensidad, que se encuentran a $44^{\circ}$ y $51.3^{\circ}$, corresponden a las reflexiones de nicromel, mientras que los picos restantes que se encuentran a $22^{\circ}, 31.4^{\circ}, 38.6^{\circ}$ y $55.9^{\circ}$, fueron indexados utilizando la tarjeta 050626 de los archivos de difracción de polvos (PDF, por sus siglas en inglés) correspondiente a la fase tetragonal del BTO.
Puede observarse que hasta una temperatura externa de $494.8^{\circ} \mathrm{C}$ no se observan picos de difracción aparte de aquellos que corresponden al nicromel, y al menos la presencia de material cristalino sólo se hace evidente cuando las muestras son tratadas externamente a $548.8^{\circ} \mathrm{C}$, ya que se observa la presencia de un pico de difracción correspondiente a las reflexiones en los planos (110) del BTO.

La figura 4 muestra los espectros de rayos- $\mathrm{X}$ correspondientes a las películas crecidas empleando temperaturas de sustrato in-situ de $374.8^{\circ} \mathrm{C}$ a $548.8^{\circ} \mathrm{C}$ durante el depósito.

En esta figura puede observarse la evolución de la fase cristalina perovskita tetragonal con el incremento de la temperatura del sustrato. Para una temperatura de $374.8^{\circ} \mathrm{C}$ la película es amorfa, mientras que a $434.8^{\circ} \mathrm{C}$ el material empieza a cristalizar, ya que aparece un pico de difracción a $31.4^{\circ}$ correspondiente a las reflexiones en los planos (110) y otro alrededor de los $38.8^{\circ}$ correspondiente a las reflexiones en los planos (111). El incremento en la temperatura resulta en el aumento de la cristalinidad de las películas, lo cual es evidente en el incremento de la intensidad de los picos de difracción, además de la aparición de un pico a aproximadamente $52^{\circ}$ que corresponde a las reflexiones en los planos (211). Es importante remarcar que, aunque a los $434.8^{\circ} \mathrm{C}$ ya aparecen picos de difracción (de baja intensidad), no significa que la totalidad del material es cristalino. Es evidente, de los difractogramas de las películas, que cuando son depositadas sobre los sustratos de nicromel, éstas presentan una fase pura sin fases secundarias (figuras 3 y 4 ).

Por otro lado, una comparación de los difractogramas de las figuras 3 y 4 , muestra que las películas creci-
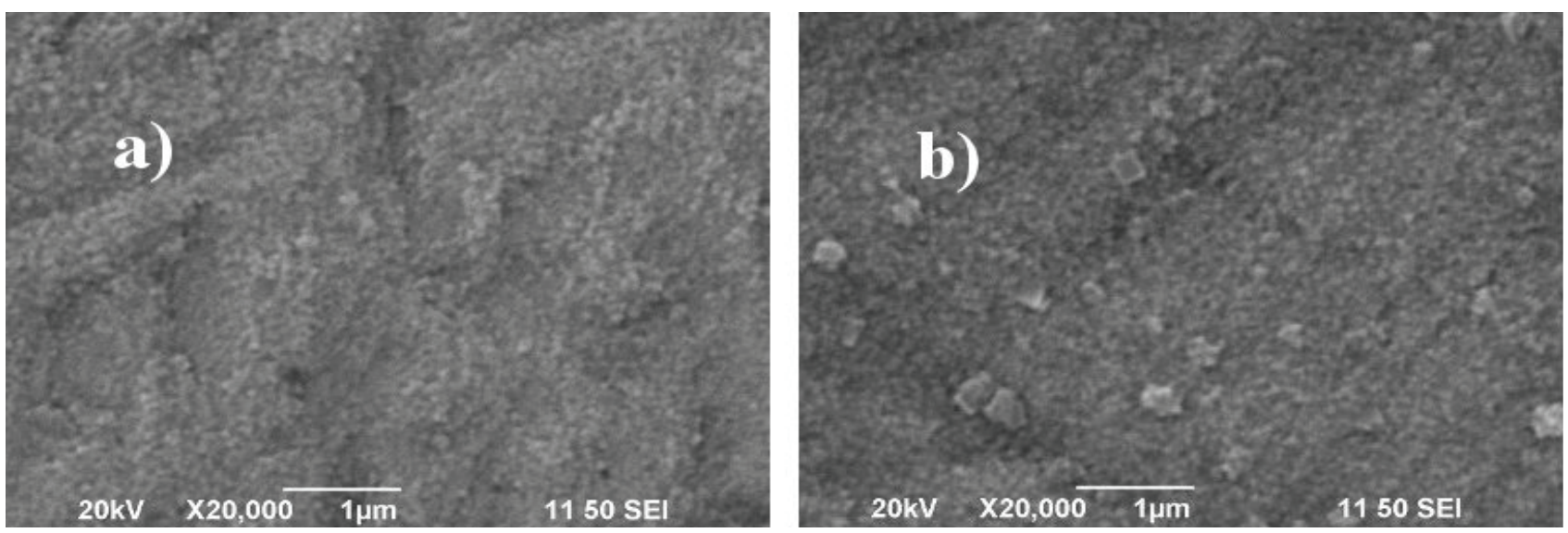

Figura 2. Micrografías de la morfología superficial de las películas delgadas de BTO depositadas sobre sustratos de Nicromel usando a) temperatura de sustrato y b) pos-tratamiento térmico a $548.8^{\circ} \mathrm{C}$ 


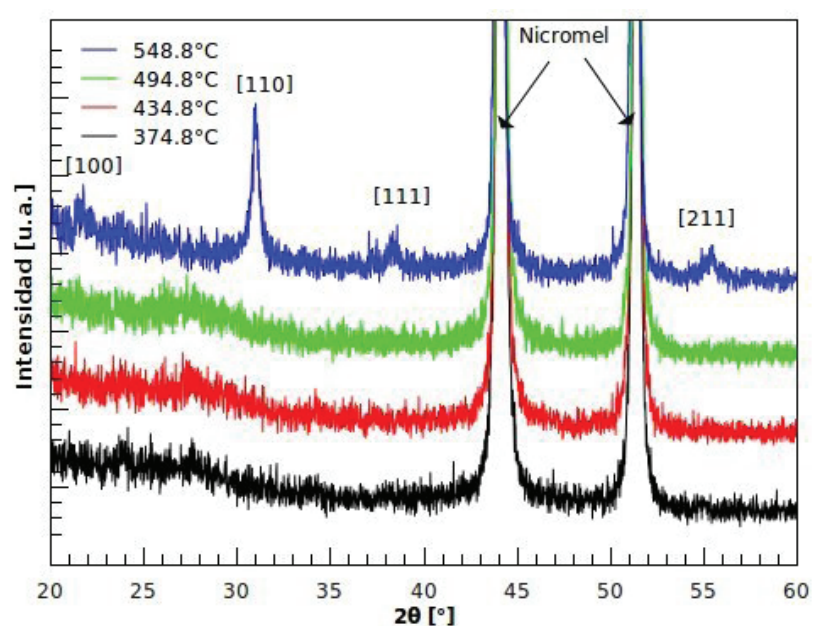

Figura 3. Difracción de rayos-X de las películas delgadas de $\mathrm{BaTiO}_{3}$ depositadas sobre sustratos de nicromel a temperatura ambiente y con tratamiento térmico externo posterior al depósito a temperaturas de 374.8 a $548.8^{\circ} \mathrm{C}$

das con temperatura de sustrato mayores al ambiente, cristalizan a temperaturas menores respecto a aquellas crecidas a temperatura ambiente y son tratadas térmicamente de forma externa a la cámara de depósito para su cristalización. Esto indica que el uso de temperatura de sustrato in-situ hace posible mejorar la cristalinidad de las películas delgadas de BTO empleando menores temperaturas. Lo anterior podría explicarse en función de la energía externa que debe suministrarse a los átomos para que éstos puedan moverse y formar una red cristalina, la cual es menor durante el proceso de crecimiento, ya que éstos cuentan con una alta energía cinética adquirida en el proceso mismo de crecimiento, además son expulsados del blanco a altas velocidades hacia el sustrato por la erosión catódica, mientras que cuando el material es tratado térmicamente posterior a su depósito por un sistema externo, toda la energía que deben adquirir los átomos para lograr su reacomodo debe suministrarse mediante el tratamiento térmico, y consecuentemente, se requiere una temperatura más alta.

Para corroborar la cristalización de las películas crecidas con temperatura de sustrato, se obtuvieron espectros de transmisión óptica y a partir de ellos, se calculó el ancho de brecha de energía prohibida $\left(\mathrm{E}_{\mathrm{g}}\right)$. La determinación del valor del $\mathrm{E}_{\mathrm{g}}$ se puede hacer empleando la aproximación de bandas parabólicas (Pasierb et al., 1998; Kirchoefer et al., 1998), para lo cual se generaron, a partir de los espectros de transmisión, curvas de $\alpha^{2}$ (donde $\alpha$ es el coeficiente de absorción) contra $h v$ (energía de los fotones incidentes). La parte lineal de estas curvas se ajustó mediante líneas rectas por el método

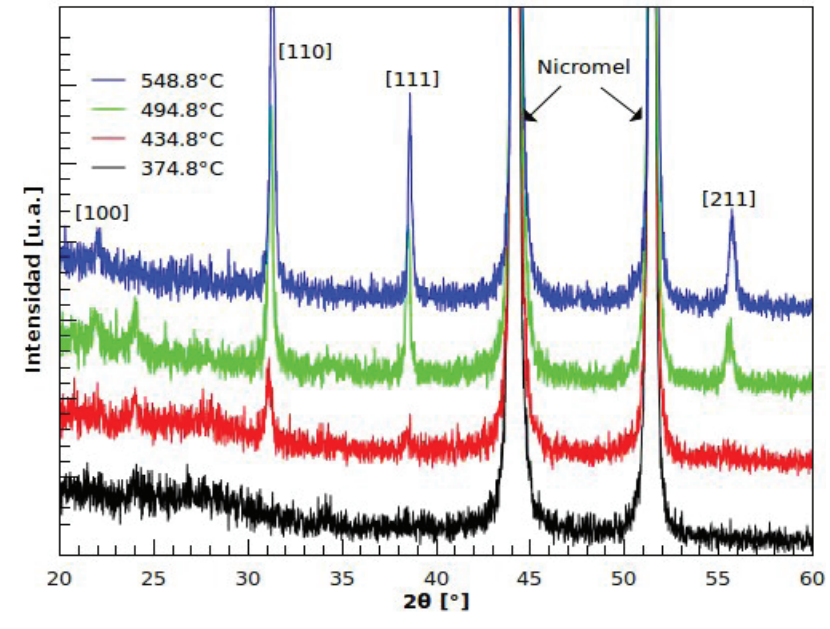

Figura 4. Espectros de difracción de rayos- $X$ de las películas delgadas de $\mathrm{BaTiO}_{3}$ depositadas sobre sustratos de nicromel

de mínimos cuadrados y su intersección con el eje de la energía dio el valor $\mathrm{E}_{\mathrm{g}}$.

Los resultados del cálculo del $\mathrm{E}_{\mathrm{g}}$ para las películas crecidas con temperatura de sustratos se grafican en la figura 5, además del $\mathrm{E}_{\mathrm{g}}$ de una muestra crecida a temperatura ambiente $\left(23^{\circ} \mathrm{C}\right)$ para referencia. Se pueden observar 3 regiones dependiendo del valor del $\mathrm{E}_{\mathrm{g}}$ obtenido. La primera con valores $\mathrm{E}_{\mathrm{g}} \geq 4.51 \mathrm{eV}$, una segunda con $\mathrm{E}_{\mathrm{g}}$ $\leq 3.9 \mathrm{eV}$ y una tercera zona intermedia, o de transición, entre las dos primeras. De acuerdo con lo reportado por otros autores (Pasierb et al., 1998; Thomas et al., 1999; Panda et al., 1998; Joshi y Desu, 1997; Thielsh et al., 1997) los valores $\mathrm{E}_{\mathrm{g}} \geq 4.51 \mathrm{eV}$ de películas de BTO corresponden a material amorfo, mientras que valores $\mathrm{E}_{\mathrm{g}} \leq 3.9 \mathrm{eV}$ son indicadores de un material cristalino. Lo anterior sugiere que para temperaturas de sustrato iguales o por debajo de los $434.8^{\circ} \mathrm{C}$ las películas son amorfas mientras que cuando la temperatura de sustrato es igual o superior a $494.8^{\circ} \mathrm{C}$ es posible obtener películas con buena calidad cristalina. Además, en la figura 6 se puede observar que no se presenta ningún valor dentro de la zona de transición, lo que sugiere que las películas no presentan fases secundarias como la cúbica. Es importante remarcar que estos resultados parecieran contradictorios a lo obtenido en el análisis de difracción de rayos-X para la película crecida con temperatura de sustrato de $434.8^{\circ} \mathrm{C}$, ya que de acuerdo con la figura 4 , existe un pico de difracción que indica la presencia de material cristalino en esta película, mientras que en la figura 5 se observa que su $\mathrm{E}_{\mathrm{g}}$ corresponde al de un material amorfo. Sin embargo, esto puede explicarse por el 


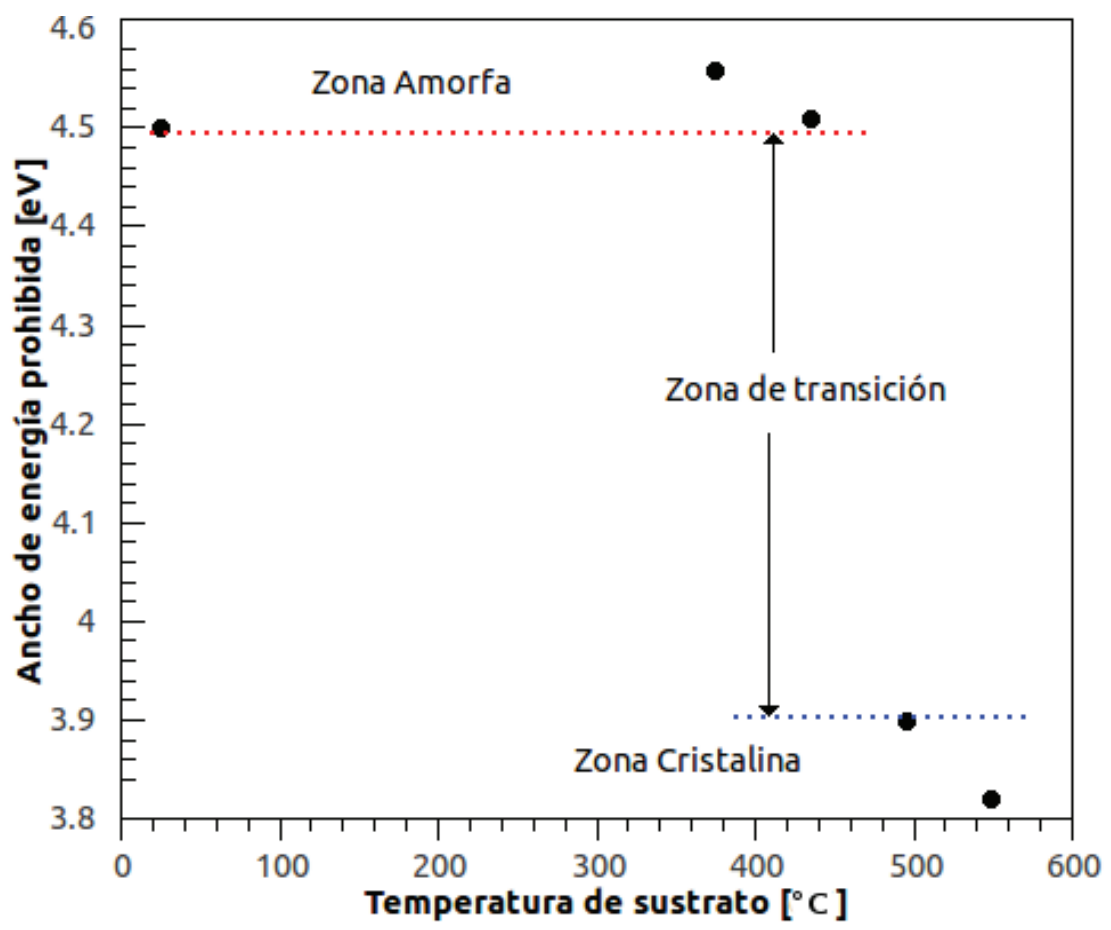

Figura 5. Dependencia del ancho de banda de energía prohibida, $\mathrm{E}_{g^{\prime}}$ en función de la temperatura de sustrato durante el depósito de las películas de $\mathrm{BaTiO}_{3}$ hecho de que en la frontera de temperaturas para la zona amorfa, los materiales crecidos son predominantemente amorfos con un bajo grado de cristalinidad (lo que origina la aparición del pico de difracción de baja intensidad como se ve en la figura 4), por lo que la luz es absorbida es su mayoría por el material amorfo, enmascarando la presencia del material cristalino y dando lugar a un $\mathrm{E}_{\mathrm{g}}$ en la región amorfa.

Por otro lado, es importante resaltar que el crecimiento de películas delgadas con temperatura de sustrato nos permite la obtención de materiales cristalinos a temperaturas por debajo de las reportadas por otros autores (Zhu et al., 2010; Pintie y Constantin, 1995; Su et al., 1997), como también lo muestra el análisis por difracción de rayos-X (figura 4).

\section{Conclusiones}

Los resultados de este trabajo mostraron que el depósito de películas delgadas de $\mathrm{BaTiO}_{3}$ usando temperatura de sustrato durante el crecimiento, permite obtener películas con buena calidad cristalina, mantener la estequiometría del material depositado, así como una buena morfología superficial de las películas. Por otra parte, el análisis del $\mathrm{E}_{\mathrm{g}}$ de las películas puede ser un indicador de la cristalinidad del material; sin embargo, como se mostró aquí, cuando se depositan películas cerca de la región de transición entre el estado amorfo y cristalino, debe ser corroborado por otras técnicas de caracterización. Es de remarcar que los resultados de esta investigación validan la utilidad del sistema de calentamiento del sustrato in-situ mencionado.

\section{Agradecimientos}

Agradecemos la asistencia técnica de Marcela Guerrero, García-Sotelo A. y Rogelio Fragoso pertenecientes al Departamento de Física del CINVESTAV-IPN. Este trabajo fue financiado por el proyecto SEP/CONACYT de Ciencia Básica 2010-2012 Núm. 165968.

\section{Referencias}

Cheng J.G., Tang J., Chu J.H., Zhang A.J. Appl. Phys. Lett, volumen 7, 2000: 1035.

Cruz M.P., Siqueiros J.M., Valenzuela J., Machorro R., Portelles J., Fundora A. Ferroelectrics, volumen 225, 1999: 319.

Hirata G.A., Lopez L.L., Siqueiros J.M. Sup. y Vac, volumen 9, 1999: 147.

Joshi P.C. y Desu S.B. Thin Solid Films, volumen 300, 1997: 289-294.

Keis V.N., Kozyrev A.B., Khazov M.L., Sok J., Lee J.S. Elect. Lett., volumen 34 (número 11), 1998: 3.

Kim S.T., Kim H.H., Lee M.Y., Lee W.J. Jpn. J. Appl. Phys A, volumen 1 (número 36), 1997: 294.

Kirchoefer S.W., Pond J.M., Carter A.C., Chang W., Agarwal K.K., Horwitz J.S. Chrisey D.B. Micr. Opt. Tech. Lett., volumen 18 (número 3), 1998: 169. 
Lee W.J., Kim Y.M., Kim H.G. Thin Solid Films, volumen 269, 1995: 75.

Márquez-Herrera A., Zapata-Navarro A. Cruz-Jáuregui M.P. J. of Mat. Sci., volumen 40, 2005: 5103-5105.

Márquez-Herrera A., Hernández-Rodríguez E., Cruz-Jáuregui M.P., Zapata-Torres M., Zapata-Navarro A. Calentador de sustratos compacto y de bajo costo para tratamientos térmicos in situ de películas delgadas depositadas por rf-sputtering. Rev. Mex. de Fis., volumen 56 (número 1), 2010:85-91.

Panda B., Dhar A., Nigam G.D., Bhattacharya D., Ray S.K. Thin Solid Films, volumen 332, 1998: 46-49.

Pintie L. y Constantin C. Dielectric and Pyroelectrric Poperties of a Ceramic Bimorph Structure, Ferroelectrics, volumen 173, 1995: 111-124.

Pasierb P., Komornicki S., Radecka M. Thin Solid Films, volumen 324, 1998: 134.

Ruckenbauer V., Hau F.F., Lu S.G., Ye-Ung K.M., Mak C.L., Wong K.H. Appl. Phys. A, volumen 78, 2004: 1049.

Shi-Jian L ., Chong-Yang X ., Xiang-Bin Z., Ji-Qun S., Bo-Fang Z. Phys. Stat. Sol. A, volumen 194 (número 1), 2002: 64.

Somekh R.E. J. Vac. Sci. Technol. A, volumen 2, 1984: 285.

Sreenivas K., Reaney I., Maeder T., Setter N. J. Appl. Phys., volumen 75 (número 1), 1994: 232.

Su Q.X., Rabson T.A., Robert M., Xiong J.X.Z., Moss S.C. Thin Solid Films, volumen 305, 1997: 227-231.
Thielsh R., Kaemmer K., Holzapfel B., Schultz L. Thin Solid Films, 1997: 203-210.

Thomas R.,. Dude D.C, Kalamasan M.N., Chanda S. Thin Solid Films, volumen 346, 1999: 212-225.

Torres-Heredia V., Muñoz-Saldaña J., Espinoza-Beltrán F.J., Márquez-Herrera A., Zapata-Navarro A. Adv. in Tech. of Mat. and Mat. Proc. J (ATM), volumen 7 (número 2), 2005: 105-110.

Zhu X.H., Defay E., Guigues B., Le-Rhu G., Dubarry C., Aïd M. J. Eur. Ceram. Soc., volumen 30 volumen 2, 2010: 471-474.

\section{Este artículo se cita:}

Citación estilo Chicago

Márquez-Herrera, Alfredo, Eric Noé Hernández-Rodríguez, Martín Guadalupe Zapata-Torres, María de la Paz Cruz-Jáuregui, Miguel Ángel Meléndez-Lira. Estudio de la cristalinidad en películas delgadas de $\mathrm{BaTiO}_{3}$ debido al tratamiento térmico. Ingeniería Investigación y Tecnología, XIV, 03 (2013): 317-323.

\section{Citación estilo ISO 690}

Márquez-Herrera A., Hernández-Rodríguez E.N., Zapata-Torres M.G., Cruz-Jáuregui M.P., Meléndez-Lira M.A. Estudio de la cristalinidad en películas delgadas de $\mathrm{BaTiO}_{3}$ debido al tratamiento térmico. Ingeniería Investigación y Tecnología, volumen XIV (número 3), julio-septiembre 2013: 317-323.

\section{Semblanza de los autores}

Alfredo Márquez-Herrera. Es investigador y egresado del Centro de Investigación en Ciencia Aplicada y Tecnología Avanzada, Unidad Legaría del Instituto Politécnico Nacional, investigador del Sistema Nacional de Investigadores, nivel I y profesor investigador del área de Ingeniería Mecánica Administrativa de la Coordinación Académica Región Altiplano de la Universidad Autónoma de San Luis Potosí.

Eric Noé Hernández-Rodríguez. Es egresado del Centro de Investigación en Ciencia Aplicada y Tecnología Avanzada, Unidad Legaría del Instituto Politécnico Nacional.

Martín Guadalupe Zapata-Torres. Es investigador y egresado del Centro de Investigación y de Estudios Avanzados del Instituto Politécnico Nacional (Cinvestav, Mérida). Es investigador del Sistema Nacional de Investigadores, nivel II y profesor investigador C del Departamento de Tecnología Avanzada del CICATA-IPN, Unidad Legaria.

María de la Paz Cruz-Jáuregui. Es investigadora y egresada del Centro de Investigación Científica y de Educación Superior de Ensenada (CICESE). Física de formación es investigadora del Sistema Nacional de Investigadores, nivel II y profesor-investigador del Departamento de Materiales Avanzados del Centro de Nanociencias y Nanotecnología (CNyN)-UNAM.

Miguel Ángel Meléndez-Lira. Es investigador y egresado del Centro de Investigación y de Estudios Avanzados del Instituto Politécnico Nacional (Cinvestav). Físico de formación, es investigador del Sistema Nacional de Investigadores, nivel III y profesor investigador 3C de Departamento de Física del Cinvestav, Unidad Zacatenco, así como profesor titular de tiempo parcial en la UAM-Atzcapotzalco. 